\title{
Labyrinthe
}

17 | 2004 (1)

Jacques Rancière, l'indiscipliné

\section{Politiques de la lecture}

\section{Renaud Pasquier}

\section{OpenEdition}

Journals

Édition électronique

URL : http://journals.openedition.org/labyrinthe/172

DOI : $10.4000 /$ labyrinthe. 172

ISSN : 1950-6031

Éditeur

Hermann

Édition imprimée

Date de publication : 15 avril 2004

Pagination : 33-63

Référence électronique

Renaud Pasquier, «Politiques de la lecture », Labyrinthe [En ligne], 17 | 2004 (1), mis en ligne le 13 juin 2008, consulté le 19 avril 2019. URL : http://journals.openedition.org/labyrinthe/172 ; DOI : 10.4000/ labyrinthe. 172

Propriété intellectuelle 


\title{
POLITIQUES DE LA LECTURE
}

\author{
Renaud PASQUIER
}

Toute pensée un peu singulière se signale par ceci qu'elle ne dit jamais fondamentalement qu'une seule chose et ne peut s'empêcher de la risquer à chaque fois dans le prisme coloré des circonstances $(P P, 13)$.

On ne prétendra pas identifier ici la seule et même chose inlassablement réaffirmée par Jacques Rancière. Il s'agit d'aborder la singularité de son œuvre par ce biais - selon nous primordial - de la lecture, comme problème et comme pratique. Tant il est vrai que c'est dans et par la lecture, à travers les échos de paroles autres, que se forge et se déploie une voix propre, celle d'un philosophe-lecteur. Non pas un commentateur. Point d'exégèse, mais la mise en œuvre d'une écoute, d'une sensibilité à des voix multiples.

\section{I - LES « ANIMAUX LITTÉRAIRES » DANS « L'ÎLE DU LIVRE »}

«Qu'est-ce que lire?» $(L C, 6)$ : Louis Althusser plaçait cette question au cour de Lire «Le Capital », travail mené avec ses élèves et appelé à un retentissement aussi considérable qu'inattendu. Ce premier acte du parcours intellectuel de Jacques Rancière ne nous intéresse pas ici en tant que tel: c'est la rupture radicale avec Althusser qui constitue le geste inaugural d'une œuvre propre, rupture avec l'«idée de la lecture qui soutient l'entreprise althussérienne » $(C M, 157)$, rupture signifiée avec virulence dans le Mode d'emploi pour une réédition de Lire « Le Capital » ${ }^{1}$, et surtout dans le premier ouvrage de Rancière, La Leçon d'Althusser ${ }^{2}$.

1. Texte prévu comme préface à une nouvelle édition complète du volume finalement publié, pour des raisons précisées dans un avertissement, par Les Temps modernes, $\mathrm{n}^{\circ} 328$, novembre 1973, p. 788-807. 2. Premier ouvrage de Rancière, en forme de réquisitoire contre son ancien maître, à partir d'une lecture de la Réponse à John Lewis, récemment parue. 
Le projet même de Lire «Le Capital » récusait la « lecture innocente » ou « religieuse » $(L C, 6)$, au profit d'une lecture symptômale: mettant au jour l'insu ou l'impensé du texte, elle « décèle l'indécelé dans le texte même qu'elle lit, et le rapporte à un autre texte, présent d'une absence nécessaire dans le premier » (23). Lire, c'est faire voir un invisible intrinsèque et essentiel - c'est démystifier. Et telle est bien la tâche de la lecture althussérienne, arme de la science contre l'idéologie. L'enjeu politique est clair: il y aurait d'un côté les brouillards de l'idéologie et ceux qui s'y perdent, de l'autre la science qui les dissipe, par l'entremise de ceux qui savent. Par-delà les pages du livre, le lecteur compétent, le savant, saurait seul la vérité de pratiques ignorée par leurs acteurs mêmes:

La vérité de l'histoire ne se lit pas dans son discours manifeste, parce que le texte de l'histoire n'est pas un texte où parlerait une voix (le Logos), mais l'inaudible et illisible notation des effets d'une structure de structure $(L C, 8)$.

Ce couple science/idéologie - recouvrant le partage savants/ignorants est la cible première de Rancière; il inscrit cette lecture qui fut sienne « dans le système des pratiques discursives du pouvoir ${ }^{3}$ », y dénonçant une posture de domination, quand bien même prétendrait-elle servir la cause de l'émancipation - et surtout pour cette raison. Ce partage ne ferait que reconduire le « beau mensonge » platonicien nécessaire à la bonne marche de la cité, celui de la distribution des âmes, d'or pour les uns, de fer pour les autres, pieux mensonge qui fonde l'harmonie, qui assure que chacun s'en tient à son affaire, sans s'aventurer au-delà de son territoire propre $^{4}$ (voir $\left.P P, 36-44\right)$. Quoi qu'ils en aient, les libérateurs autoproclamés, chantres d'une lecture du soupçon et du dévoilement, sont donc désignés comme héritiers de Platon l'antidémocrate, le philosophe « totalitaire », dont la fiction - revendiquée comme telle par Socrate - n'a d'autre but que d'assurer au philosophe le privilège de la pensée. Opération répétée donc par les démystificateurs modernes ${ }^{5}$, qui, à rebours de Platon, prétendent subordonner l'émancipation à la démystification.

3. Mode d'emploi..., art. cit., p. 806.

4. La République III, 415a.

5. En sus d'Althusser, on citera les figures du Philosophe et ses pauvres, Marx, Sartre, et surtout Bourdieu, cible répétée des attaques de Rancière et des Révoltes logiques, notamment dans le volume collectif L'Empire du sociologue, entièrement consacré à cette critique de Bourdieu. 
Dénoncer cette fiction, rejeter le partage, refuser un mode de lecture, c'est tout un ; la réflexion propre de Rancière s'élabore à partir de cette rupture, accusée, approfondie, enrichie ${ }^{6}$. Les personnages qui peuplent ses textes sont d'abord des lecteurs: avatars d'Althusser, lecteurs agréés, professionnels, dont Pierre Bourdieu est longtemps l'incarnation la plus récurrente; lecteurs hérétiques surtout, contrebandiers des mots, à qui les livres ne sont pas destinés, les héros de $L a$ Nuit des prolétaires, le menuisier Gauny, Claude Genoux, mais aussi Emma Bovary, Véronique Sauviat-Graslin, et bien sûr Don Quichotte, mauvais lecteur s'il en est. À la lecture-confiscation des premiers s'oppose une lecture-appropriation des seconds. Appropriation mutuelle: car si ces parvenus s'emparent des mots et de la culture des autres, s'ils transgressent la frontière interdite, ces mots à leur tour se saisissent d'eux et les arrachent à leur destinée, interdisant toute assignation stable à ces êtres qui rêvent d'ailleurs.

À travers l'étude patiente et minutieuse de ces figures transgressives, Rancière met en place une anthropologie minimale et paradoxale aux accents aristotéliciens ${ }^{7}$ : « êtres parlants », les hommes sont tout autant « êtres lisants », des animaux littéraires.

L'homme est un animal politique parce qu'il est un animal littéraire, qui se laisse détourner de sa destination « naturelle » par le pouvoir des mots $(P S, 63)$.

La lecture apparaît ici comme l'activité humaine par excellence, précisément en ce qu'elle contrecarre toute naturalisation de l'humanité. En outre, elle ne se définit aucunement sur le mode de l'avoir, comme accumulation du savoir, mais comme une opération venant perturber l'être du lecteur - ou la perception qu'il en a, ce qui revient au même. Reste à comprendre comment procède ce détournement, et ce que Rancière entend par l'adjectif littéraire.

6. « Don Quichotte, Althusser et la scène du texte » (voir $C M$ ) vient l'attester: texte étonnant qui revient sur Althusser, dans un livre consacré avant tout à la littérature. Le ton polémique de 1974 a laissé place à une distance respectueuse, mais les conclusions sont les mêmes. Il marque aussi le retour d'une pensée sur elle-même, reconsidérant l'origine de son parcours à la lumière de ses derniers développements. Que cela se fasse dans le cadre d'une réflexion sur la lecture n'est pas un détail à dédaigner.

7. Lire les précisions de Rancière sur son usage d'Aristote, dans l'entretien « Histoire des mots, mots de l'histoire », Communications n 58, 1994, p. 92. 
Cette littérarité qui définit la condition humaine n'a guère de rapport avec la literarurnost des formalistes russes mise au goût du jour par la théorie littéraire ${ }^{8}$. Loin de signifier une essence de la littérature, littérarité désigne chez Rancière un régime des rapports entre corps et mots, défini comme disponibilité mutuelle des uns aux autres: d'une part des corps prêts à accueillir toute fiction, à l'élever au rang de vérité; d'autre part des énoncés errants, sans destinataire privilégié. Aux figures de lecteurs hérétiques correspond une image platonicienne du livre; ces blocs de mots sans origine ni but repérables, c'est en effet dans le Phèdre que nous les rencontrons, où Socrate raconte le mythe de l'invention de l'écriture par le dieu Theuth, doublement mise en accusation par le roi Thamous: comme parole orpheline, à la fois muette (sans père pour la défendre - pour en fixer le sens) et bavarde (puisqu'elle s'adresse à tous, sans établir de relation personnelle avec un lecteur particulier). Or, c'est une certaine forme de communauté que dessine cette parole errante et indéterminée, où aucun corps (aucun lecteur) n'est privilégié - soit une communauté fondée sur l'égalité, en un mot: la démocratie, honnie par Platon.

Le fil de la lecture, que nous avons suivi depuis la critique de l'althussérisme, vient ici nouer politique et littérature dans une même perspective an-archique ${ }^{9}$ : l' abolition des « castes de lecteurs » rencontre une pensée de la démocratie, empruntée à l'antidémocrate Platon ${ }^{10}$, comme mode de circulation des mots (et non comme institution), mais aussi l'idée même de littérature telle qu'elle s'invente à l'aube du XIX ${ }^{\mathrm{e}}$ siècle sur les ruines de l'ancienne hiérarchie générique des « belles-lettres », parole muette et bavarde, sans garant en amont de

8. La distinction entre les deux usages du terme est soulignée par Solange Guénoun dans l'entretien qu'elle mène aux côtés de John H. Kavanagh: "Literature, Politics, Aesthetics: Approaches to Democratic Disagreement », Substance, n 92, 2000, p. 7-8.

9. Voir «L'inadmissible », où l'articulation littérature/politique est élaborée autour de cet « anarchisme » $(A B, 189$ et 194$)$.

10. Platon verrait «plus juste dans les fondements de la politique et de la démocratie que les tièdes amours de ces apologistes fatigués qui nous assurent qu'il convient d'aimer " raisonnablement », c'est-à-dire «modérément » la démocratie » (Més., 29). Plus encore, il est la pierre de touche pour toute tentative philosophique: «Peut-être notre situation philosophique est-elle bien figurable ainsi : nous voyons s'y opposer diverses manières de choisir Platon contre Platon » $(A B, 180)$. Si Rancière reprend là une formule de Jean Borreil, on entend aussi l'écho de Michel Foucault: «Et si, à la limite, on définissait philosophie toute entreprise, quelle qu'elle soit, pour renverser le platonisme? » Foucault, « Theatrum philosophicum », Critique n² 282, novembre 1970, p. 885-908. 


\section{Politiques de la lecture}

ses trajets, sans destinataire désigné en aval; Rancière rappelle que l'absolu romantique, celui du style flaubertien, n'est rien d'autre, étymologiquement, que la déliaison $(P M, 107)$. Ce point d'intersection en une commune (mais non identique) délégitimation des hiérarchies, une formule le désigne, récurrente chez Rancière: "l'île du livre ». Qu'entendre par cette insularité du livre ? Son irréductibilité aux circuits légitimes de communication et de domination, par lui entravés. Ce qui en fait la cause de ces déclassements - ruptures proprement dites avec le «tissu social »-dont les victimes sont préci-

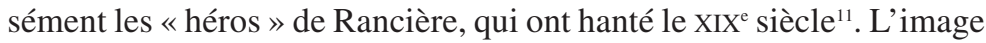
îlienne éclaire ainsi l'autre face de la littérarité, à savoir les modalités de saisie des mots par les lecteurs. Joseph Jacotot, figure centrale du Maître ignorant, où apparaît l'expression, fait du livre la clef de voûte de son "enseignement universel », fondé sur le présupposé de l'égalité des intelligences ; les lecteurs-voyageurs qui le suivent partent dans l'île se soumettre à cette puissance d'interruption qu'ils actualisent.

La métaphore du détournement, évoquée plus haut pour désigner l'effet des mots sur les corps, pouvait induire un rapport de séduction, voire de fascination: une rhétorique habile transformerait les vessies en lanternes dans des esprit naïfs. Il n'en est rien: la lecture est un exercice de l'intelligence, non une manifestation de la crédulité. Jacotot regarde la confrontation entre livre et lecteur comme une rencontre entre deux intelligences, une procédure de vérification de leur égalité, dont le livre se fait le support matériel. Ainsi ses élèves hollandais apprennent-ils le français sans autre aide qu'une édition bilingue du Télémaque de Fénelon et les instructions minimales de Jacotot: apprendre les mots du livre, les répéter, les réutiliser à d'autres fins.

Tout s'était joué par force entre l'intelligence de Fénelon qui avait voulu faire un certain usage de la langue française, celle du traducteur qui avait voulu en donner un équivalent hollandais, et leur intelligence d'apprentis qui voulaient apprendre la langue française $(M I, 20)$.

11. Le Curé de village de Balzac - qui place une Bovary avant la lettre au cœur d'une intrigue dénouée dans une île - sert à Rancière de fable exemplaire de ce trouble démocratique attaché à l'insularité livresque. Voir « Balzac et l'île du livre » $(C M, 115-136)$. 
Lire, c'est traduire: reformuler un texte observé, appris et répété. Et hors de ce texte, point de recours; on ne peut fuir le face-à-face avec le livre ${ }^{12}$, l'on doit puiser en lui seul les ressources nécessaires comme Robinson dans son île. L'émancipation s'oppose donc radicalement à l'explication, qui procède de l'intelligence supérieure du maître, seul apte à révéler ce que cachent les mots du livre; or

il n'y a rien derrière la page écrite, pas de double fond qui nécessite le travail d'une intelligence autre, celle de l'explicateur; pas de langue du maitre, de langue de la langue dont les mots et les phrases aient pouvoir de dire la raison des mots et des phrases d'un texte $(M I, 20)$.

On ne saurait imaginer position plus éloignée de celle d'Althusser, critique plus radicale de la démystification - quand bien même il faut maintenir la distinction entre Rancière et Jacotot: où ce dernier proclame l'impossibilité de donner corps à l'égalité, Rancière invoque la forme du tort, principe du litige qui rend effectif le trait égalitaire (voir Més., 58-59).

Résumons : la lecture hérétique, que Rancière oppose à la démystification inégalitaire, est un acte d'intelligence qui perturbe le cours normal des mots, permettant par là de refuser une condition, une position dans ce circuit de paroles. Mais détacher ces lecteurs de leur identité, ce n'est évidemment pas leur donner une nouvelle place dans la chaîne, fût-elle plus noble, ni les rendre à une individualité supposée plus authentique. Il semble qu'aux attaches sociales se substituent d'autres liens, frêles jusqu'à l'évanescence, soit une communauté, inconsistante, de ceux qui « reliés par le seul trajet des lettres sans corps $[\ldots]$ ne se voient pas et ne voient pas celui qui est écrit ${ }^{13} »$, une communauté qui ne remplace pas la construction sociale concrète, mais s'y superpose - selon la logique dite de «l'un-en-plus », celle de la littérature comme de la démocratie $(A B, 189-201)^{14}$. Les lectures transgressives créent ainsi l'étrange " communauté des gens seuls », « qui ne connaît que des singuliers », et qu'on nommera aussi

12. C'est de fait la seule tâche du maître: exercer sa volonté, enfermer l'apprenti dans le livre en lui interdisant toute sortie (voir $M I, 24-28$ ).

13. «La Chair des mots », Po\&sie, n ${ }^{\circ} 77,1996$, p. 80-92.

14. Voir aussi «La raison de la mésentente » (Més., 71-91). 


\section{Politiques de la lecture}

« communauté des égaux ${ }^{15}$ ». On est en droit de s'interroger sur le mode d'existence de telles formes de l'être-ensemble: Rancière s'attache à répondre en identifiant démocratie et dissensus (voir « Démocratie ou consensus », Més. 135-165).

En dehors de l'épineux problème de la communauté, deux autres questions surviennent quant à la matérialité même de la lecture. À propos du livre, d'abord, qui demeure une forme, Télémaque n'étant guère envisagé par Jacotot (et Rancière) dans sa singularité: quels livres donc, et comment entravent-ils le continuum discursif? Plus déroutante encore, l'absence remarquable dans Le Maître ignorant et ailleurs - de toute description de l'acte de lecture, sinon par synonymie (« reformulation », «traduction ») où il n'apparaît que comme déchiffrement. Qu'il n'y ait trace d'une quelconque méthode ne nous étonnera certes pas, qui serait bien incongrue ici; c'est plutôt la mise en question de la lecture comme processus qui nous paraît manquer dans ces représentations où on la perçoit comme un rapport immédiat du lecteur et du livre - quelles que soient les difficultés rencontrées par le premier pour s'approprier le second. Aussi le plus simple est-il de se tourner vers les pratiques du lecteur qu'est Rancière, et d'envisager comment il articule cette pensée de la lecture avec son approche des textes.

\section{II - RANCIÈRE LECTEUR}

Pas de sympathie chez Rancière à l'endroit de cette

prolifération de textes pour apprendre à lire les livres, c'est-à-dire pour les rendre illisibles, de paroles propres à rendre toute voix inaudible, le triomphe des explicateurs et des - logues en tout genre ${ }^{16}$.

Si l'on ne trouvera nul mode d'emploi chez lui, il est néanmoins possible de dégager des constantes, des partis pris récurrents qui constituent un ensemble de protocoles identifiables, jamais exemplaires ou prescriptifs.

15. Voir « La communauté des égaux » $(A B, 129-174)$.

16. «Vie intellectuelle: l'événement de 1968 », La Quinzaine littéraire, n 459, 1986, p. 35-36. 


\section{Paraphrase/Rephrasage}

En un sens, le lecteur Rancière se contenterait de mettre rigoureusement en œuvre les principes prônés par Jacotot, qui élève l'attention au rang de vertu cardinale. De même ne s'agit-il jamais de déceler un hypothétique «double fond », mais de rendre compte fidèlement de la pensée qui s'exprime dans les mots. Pas question, bien sûr, de fournir un équivalent conceptuel de l'œuvre, qui n'est pas appréhendée comme structure, encore moins comme émanation d'un temps, d'un être pur du langage ou d'un individu socialement situé. L'œuvre est un geste - ou plutôt un ensemble organisé de gestes. La pensée se manifeste par une série d'opérations ${ }^{17}$, par une certaine disposition ${ }^{18}$. L'auteur, tête de turc de tant de théoriciens, n'est ici ni absent ni mort; mais aucun privilège ne lui est accordé en tant que tel. Aucune construction biographique n'en fera le dépositaire du sens ${ }^{19}$, il n'est pas dissociable de l'acte de parole qui est aussi acte d'intelligence. Rancière échappe à l'opposition structure/sujet en posant un sujet non antécédent à l'acte, qui n'existe que pour et par lui. Conception pragmatique et dynamique, pour laquelle l'œuvre est événement ${ }^{20}$, ou subjectivation:

Par subjectivation on entendra la production par une série d'actes d'une instance et d'une capacité d'énonciation qui n'étaient pas identifiables dans un champ d'expérience donné, dont l'identification va de pair avec la refiguration du champ de l'expérience ${ }^{21}$ (Més., 59).

17. «La poésie en général est une opération de pensée qui demande à être pensée comme telle », Le Millénaire Rimbaud, préface, 1993, p. 5.

18. «Il dispose les mots de sa pensée de sorte qu'ils rompent le cercle ordinaire du banal et du caché » (Mall., 10)

19. Voir à ce propos les commentaires de Rancière sur Rimbaud le fils, de Pierre Michon, dans « Rimbaud: les voix et les corps » (CM, 62-64).

20. « On supposera [...] qu'un événement de pensée singulier porte la poésie de Rimbaud. [...] Cet événement, c'est l'accrochage du roman familial et de son latin d'enfance sur une autre légende et une autre musique, la grande musique du XIX ${ }^{\mathrm{e}}$ siècle » $(C M, 64)$.

21. Sur la subjectivation, lire la première des « Dix thèses sur la politique » $(A B, 223-226)$. Point de contact entre littérature et politique, cette notion marque aussi l'irréductibilité de ces deux opérations dissensuelles, puisque la subjectivation littéraire va en quelque sorte « trop loin » dans la désidentification pour permettre une quelconque scène du litige. Voir « Le malentendu littéraire », dans Le Malentendu, Puv, 2003, et l'entretien avec François Noudelmann, « La communauté comme dissentiment », Rue Descartes n 42, 2003, p. 96. 


\section{Politiques de la lecture}

Où s'affirme aussi un critère de départ entre les livres: ceux qui font événement et les autres; toutes autres distinctions s'effacent devant celleci (philosophie et littérature, discours savant et parole vulgaire, etc.).

Avant de revenir sur cette pensée de l'événement-subjectivation, considérons à quoi engage la fidélité requise par telle lecture. Elle est moins interprétation qu'explicitation, presque la paraphrase honnie des professeurs de français. Le lecteur peut s'y faire archiviste: le travail d'édition, pratiqué dans La Nuit des prolétaires, L'archive ouvrière, et Louis-Gabriel Gauny, le philosophe plébéien, s'inscrit de fait dans le prolongement de celui d'explicitation où le philosophe s'efface derrière le texte; un effacement que Rancière, dans Le Maître ignorant, pousse au point où sa voix se confond avec celle de Jacotot. Manière sans doute de « ne pas prendre ceux dont [il parle] pour des imbéciles, qu'ils [soient] poseurs de parquets ou professeurs d'université » $(P P, 13)$, et de vérifier le présupposé égalitaire, en faisant entendre des voix oubliées. Telle modestie expose pourtant à une objection althussérienne: Rancière n'aurait-il pas opéré trop large virage, jusqu'à coïncider avec les cibles de son ancien maître, ces lecteurs naïs, religieux, qui font « d'un discours écrit la transparence immédiate du vrai » $(L C, 7)$ ? Pour qui traque aussi opiniâtrement les discours du propre, il serait étonnant de prétendre à une restitution objective des textes dans leur vérité pure. De fait, il n'y a pas chez Rancière d'objectivation, et la paraphrase évoquée plus haut se complique en rephrasage:

Il est clair que tout mon travail théorique a essayé de parler à travers les paroles des autres, de faire parler autrement les paroles des autres en les « rephrasant », en les remettant en scène ${ }^{22}$.

La métaphore théâtrale complique la prétention restitutive. Le lecteur, que l'on disait effacé, en est rehaussé. Surtout l'adverbe nous intéresse: c'est bien autrement que doivent parler les paroles des autres, et non dans leur illusoire pureté originelle.

\footnotetext{
22. Entretien accordé au site Arje, réalisé par Andrea Benvenuto, Laurence Cornu et Patrice Vermeren
} à Paris le vendredi 24 janvier 2003. 


\section{Rectifications/Reviviscences}

On peut comprendre l'adverbe d'une deuxième manière, en référence, non à l'introuvable nudité première de la parole, mais aux voiles qui l'ont recouverte et rendue inaudible, posés par des générations de lecteurs. Au-delà donc de la (pas si) simple explicitation, la spécificité des approches de Rancière réside dans leur caractère second: re-lectures, et non lectures, elles visent - au sens propre - tout autant, sinon plus, un lecteur antérieur que le texte lui-même. Leur ambition est toujours polémique, et rectificatrice:

Certains noms projettent une ombre qui les dévorent. [...] Les pages qui suivent voudraient aider à débrouiller cette nuit, à dégager de l'ombre portée des mots de poète et d'obscurité la difficulté propre de Mallarmé (Mall., 7-8).

La prose de Rancière abonde en ces tournures ironiques qui viennent démanteler la doxa la plus assurée, stigmatiser un survol trop rapide, une conclusion hâtive; à propos de Don Quichotte:

La réponse immédiate dit que la folie consiste à ne pas savoir distinguer réalité et fiction, à prendre l'une pour l'autre. [...] C'est en somme l'explication que nous propose Cervantès. [...] Le problème est que le même Cervantès nous offre plusieurs épisodes qui n'entrent pas dans ce schéma ${ }^{23}$.

Ou bien, en considérant la fameuse lettre de Flaubert à propos du « livre sur rien »:

Cette lettre est « bien connue », suffisamment pour qu'on se dispense généralement d'être attentif aux termes du problème $(P M, 105)$.

La rectification consiste à mettre à mal ce soi-disant bien connu, ce qu'un peu d'attention permet de mener à bien. Simples remontrances de professeur intransigeant? Il s'agit surtout de délier les textes des dispositifs conceptuels qui les attachent à une place et une fonction univoques et déterminées. Don Quichotte, loin de réaffirmer par ses déconvenues le partage réalité/fiction, vient au contraire le brouiller en

23. «La Chair des mots », dans Po \& sie, p. 87; c'est moi qui souligne. 


\section{Politiques de la lecture}

lui opposant la vérité du livre, qu'il se propose d'attester dans sa chair, fou par devoir et non par confusion ${ }^{24}$; le style comme «manière absolue de voir les choses » défendu par Flaubert ne relève aucunement de l'art pour l'art, et signifie au contraire

manière de voir les choses telles qu'elles sont, dans leur «absoluité » $[\ldots]$ déliées des formes de présentation des phénomènes et de liaison entre les phénomènes qui définissent le monde de la représentation $(P M, 107)$.

L'exigence rectificatrice justifie dès lors le corpus de Rancière, de manière évidente dans le domaine littéraire: Flaubert, Mallarmé, Proust, soit la sainte trinité de la modernité, hérauts de l'absolu littéraire, en somme les «piliers » du canon, désencastrés par Rancière. À première vue, les travaux d'édition, l'invention d'archives, qui par définition exhument des textes jamais lus, ne paraissent pas obéir à ce principe de contre-lecture; ils tiennent pourtant de la même réaction à une distribution prétablie des lieux, en l'occurrence au modèle historien d'une appartenance organique à un temps déterminé à quoi sont vite réduites les voix des prolétaires. Propre de l'art, propre du corps (lectures biographiques ou sociologiques), la cible est la même, par-delà l'apparente diversité des discours appréhendés; toujours le même « verrouillage », la même «prise d'otage » des textes et des paroles, qui les scelle sans recours à un lieu propre. Charge à Rancière de les " libérer ${ }^{25}$ ", de leur rendre ce caractère flottant et aléatoire des phrases orphelines du Phèdre.

Entendons-nous sur ce verbe, « rendre »: la fin de telles lectures, on l'a déjà dit, n'est pas la restitution d'un sens authentique (qui relèverait du propre); c'est celle d'une efficace, d'une vigueur polémique. Raviver les braises cachées sous la cendre des commentaires ${ }^{26}$. Réveiller une capacité provocatrice depuis trop longtemps ensevelie: un menuisier qui philosophe (Gauny), ou un poète du pur langage qui se préoccupe de politique (Mallarmé) sont des figures transgressives, qui viennent troubler un ordre établi - ou plus exactement « refigurer

24. L'analyse complète se déploie dans « Le corps de la lettre: Bible, épopée, roman » $(C M, 104-$ 110).

25. Millénaire Rimbaud, p. 6-9.

26. Alain Badiou explicite fort bien ce traitement appliqué à « des strates d'énoncés abolis ou détournés. [Rancière] se propose d'en faire à nouveau circuler l'énergie signifiante ». Abrégé de métapolitique, Paris, Seuil, coll. « L'ordre philosophique », 1998, p. 122. 
le champ de l'expérience », puisqu'il s'agit bien de s'attaquer à un consensus, c'est-à-dire un système d'évidences sensibles. D'où le recours incessant à Platon, dont Rancière loue « la force provocante » et «l'extraordinaire franchise » $(P P, 83)$, à mille lieues des modernes « processus d'euphémisation » de nos sociétés (Més., 51); sa virulence antidémocrate réveille le scandale originel du mot « démocratie »: synonyme moderne de consensus, il en devient, retrempé dans la phrase platonicienne, le parfait antonyme. La lecture, véritable décapage, régénère les vieux mots fatigués - ou déclarés tel ${ }^{27}$. Et c'est dans leur passé que l'on en puise un usage nouveau.

\section{Connexions}

Redonner aux phrases leur jeunesse, leur offrir une possibilité d'agir dans le temps du lecteur: il en va de l'historicité des textes. Les libérer de l'assimilation à une position, soit à des coordonnées socio-historiques, c'est déclarer la guerre à un certain historicisme. Bourdieu fait ici encore figure d'adversaire privilégiée ${ }^{28}$, qui tonne contre les lectores reconduisant la « neutralisation déréalisante ${ }^{29}$ » d'une lecture occultant la place de l'auteur dans le jeu social. On aurait pourtant tort de voir en Rancière un chantre de la déshistoricisation, de cette lecture «pure » vilipendée par Bourdieu: la préface de La Politique des poètes est à ce titre très instructive, où Rancière met en cause la lecture d'Hölderlin par Heidegger qui

[enferme] le poète dans la fonction religieuse et oraculaire du gardien de l'origine,

ce qui est

méconnaitre la force propre et la teneur historique du mouvement de pensée de Hölderlin ${ }^{30}$.

27. Voir « Les gros mots », introduction des Scènes du peuple, récent volume rassemblant les articles parus dans les Révoltes logiques.

28. La critique de la lecture "pure », engagée dans Les Règles de l'art est développée dans la deuxième partie des Méditations pascaliennes, « Les trois formes de l'erreur scolastique », p. 63110, en particulier dans la « Digression » des p. 75-80, « Critique de mes critiques », et dans le « PostScriptum », p. 101-109, « Comment lire un auteur. » Les Méditations pascaliennes, Paris, Seuil, coll. « Liber », 1997; Les Règles de l'art. Genèse et structure du champ littéraire, Paris, Seuil, 1992.

29. Méditations pascaliennes, op. cit., p. 101.

30. La Politique des poètes, p. 13. 


\section{Politiques de la lecture}

De fait, la force polémique des mots - à mettre au jour - n'intervient que dans une configuration historique précise. Où réapparaît la notion d'événement, abordée plus haut. La poésie de Rimbaud est "événement de pensée » pour autant qu'elle est « légende du siècle » $(C M$, 63), qu'elle « peut mettre le siècle dans la disposition du poème », ce qui veut dire « tracer la ligne qui unit ses signifiants et ses emblèmes dispersés » (64-65); Mallarmé est salué pour « [sa] conscience aiguë de la complexité d'un moment historique » (Mall., 12-13). Il ne s'agit pas pour autant de faire de leurs œuvres des documents, témoignages exemplaires des caractères d'une époque; soit en termes bourdieusiens, des «exemplifications », doublant le discours conceptuel du sociologue $^{31}$. Cette rigueur et cette justesse dans l'écriture d'une époque supposent nécessairement une distance à son égard, un retrait qu'il faut bien nommer anachronisme ${ }^{32}$. En somme, la véritable «illusion », c'est identifier des textes à un temps, auquel, précisément, ils n'appartiennent pas au sens strict du terme. Qu'ils viennent perturber, traverser, en ouvrant d'autres temporalités - où l'on retrouve la puissance d'interruption associée à « l'île du livre». Cette inappartenance, où Bourdieu ne voit que dénégation du social, c'est donc ce à quoi ils tendent, et cela même qui fait leur prix ${ }^{33}$. Par temps, on entendra donc un tissu de paroles, un écheveau compact de mots et d'idées récurrentes dessinant les contours de légitimités. Deux remarques corollaires à cette appréhension des textes. La nécessaire distinction entre intemporel et anachronique d'abord, dont ne se préoccupe pas Bourdieu: les textes ne sont pas hors du temps, mais hors de ce temps auquel on les assimile, et qu'ils viennent bouleverser; d'où il ressort qu'il n'y a de pensée à proprement parler qu'illégitime ${ }^{34}$. La seconde remarque

31. Bourdieu affirme certes ne pas regarder l'œuvre comme « document», ce qui serait « laisser échapper la spécificité du travail littéraire », Les Règles de l'art, p. 69-70, n. 127. Aussi lui reconnaît-il une capacité de «manifestation limitée d'une vérité ». Mais les « euphémismes » de ces « exemplifications » restent nécessairement en deçà de l'objectivation pratiquée par le discours scientifique (p. 69 et 541).

32. Voir, sur ce point essentiel, «Le concept d'anachronisme et la vérité de l'historien », dans L'Inactuel $\mathrm{n}^{\circ}$ 6, 1996.

33. Sans doute Bourdieu loue-t-il la force « révolutionnaire » de Flaubert ou Baudelaire, et entendil également les arracher à la gangue de commentaires qui les étouffe. Mais il s'agit toujours en dernière instance de les rapporter à un espace social dont ils ne peuvent s'abstraire, quand bien même ils le « révolutionneraient ».

34. Rancière l'affirme dans un entretien avec Davide Panagia, «Dissenting Words: A Conversation with Jacques Rancière », Diacritics, 2000, p. 121. 
concerne ledit bouleversement: le geste, l'événement de pensée en quoi consiste l'œuvre, Rancière le figure, on l'a vu, par un tracé qui relie divers points significatifs d'un temps; mais tracer veut dire ici trancher, en une intervention singulière et locale.

Restituer l'agir propre des phrases n'est assurément pas chose évidente, dès lors qu'il s'agit de les déplacer en un autre temps. Il faut en passer par un traitement singulier exercé sur elles, second volet du « rephrasage », pendant du travail de déliaison, et son inverse: détachés des liens qui les clôturaient, les énoncés ne s'isolent pas dans une étrange solitude; ils se voient proposer des articulations nouvelles mais non consubstantielles, fragiles, qui n'entravent pas leur labilité - on retrouve le travail de déliaison/rattachement observé plus haut à propos des lecteurs. La rigidité monumentale fait place à la légèreté de mobiles structurés autour de connexions multiples, que l'on distribuera en deux types, selon une double détermination du temps : comme époque, bien sûr, mais aussi, et d'abord, comme chronologie propre de l'œuvre. Les deux partis pris de lectures revendiqués dans la préface du Philosophe et ses pauvres identifient ce double travail de connexion:

A.

Je n'ai pas déféré à la bienséance qui, chez tel ou tel auteur, distingue les œuvres reconnues ou reniées, fait la part des circonstances, comme celles de la jeunesse ou de la maturité.

Althusser fait encore ici les frais de l'ironie de Rancière ${ }^{35}$. Mais audelà de la simple pique, c'est un certain rapport aux textes que cette phrase revendique. Ceux-ci sont bien appréhendés comme œuvres, soit comme totalités cohérentes. Mais cohérence n'est pas cohésion: aucune structure contraignante n'empêche de relier tout élément de ces totalités avec tout autre - au détriment de la dimension évolutive de la démarche. De fait, la lecture, jamais réellement intégrale (même dans le Mallarmé, seule étude d'auteur à proprement parler), met en place un travail de connexions internes, articulant divers fragments d'une

35. La « coupure épistémologique » théorisée dans Pour Marx, sépare ainsi irrémédiablement le « jeune Marx », le philosophe des Manuscrits de 1844, en proie à l'idéologie, du Marx scientifique auteur du Capital. Voir Pour Marx, Paris, Maspéro, coll. « Théorie », 1965. 
œuvre: «Flaubert », chez Rancière, c'est une collection d'extraits de la correspondance, de Madame Bovary, et de La Tentation de saint Antoine; Balzac est présent à travers quelques phrases de "l'Avantpropos à La Comédie humaine » d'une part et l'intrigue du Curé de village d'autre part; Don Quichotte se résume à quelques épisodes, Maître Pierre le marionnettiste, la lettre à Dulcinée, etc. On tend vers la rhétorique de morceaux choisis. Mais ces petites anthologies sont gouvernées par un principe structurant : déniant toute prétention d'exhaustivité, elles construisent une intervention limitée à un foyer circonscrit. L'important est de rendre visible un dispositif de pensée et d'introduire un grain de sable dans une mécanique conceptuelle, aggraver une faille dans un discours de propriété. La vigilance du penseur peut fort bien s'exercer dans une tout autre perspective, selon les nécessités du moment ${ }^{36}$. Locale, l'opération s'inscrit néanmoins dans une perspective plus globale: l'œuvre appréhendée est synthétisée en un geste sténographié par la lecture. Se dessine le portrait d'une pensée, portrait dynamique, complexe, inachevé, où plusieurs profils esquissés apparaissent simultanément, difficilement compatibles.

C'est qu'il ne s'agit pas seulement de démanteler les attaches qui emprisonnent l'œuvre, mais de la toucher en ses charnières, en ses failles singulières.

Comprendre un penseur, ce n'est pas venir cö̈ncider avec son centre. C'est, au contraire, le déporter, l'emporter sur une trajectoire où ses articulations se desserrent et laissent un jeu. Il est alors possible de défigurer cette pensée pour la refigurer autrement, de sortir de la contrainte de ses mots pour l'énoncer dans cette langue étrangère dont Deleuze, après Proust, fait la tâche de l'écrivain ${ }^{37}$.

La lecture-traduction, où convergent déplacement et paraphrase, cela se trouvait déjà dans Le Maître ignorant. Mais ce travail de l'œuvre dans ses articulations nous entraîne au-delà de la simple opération de restitution ou de reviviscence. D'autres pages consacrées à

\footnotetext{
36. Rancière prend toujours soin de rapporter ses travaux à un contexte particulier. Voir par exemple les deux préfaces de La Nuit des prolétaires, à l'édition originale de 1981, puis à la réédition de 1997, ainsi que celle d'Aux bords du politique.

37. «Existe-t-il une esthétique deleuzienne? », dans Gilles Deleuze, une vie philosophique, 1998, p. $525-536$.
} 
Deleuze $^{38}$ tendent explicitement à cette mise en évidence d'une distorsion, écart à soi-même intrinsèque à l'œuvre, son prix, aussi :

La force de toute pensée forte est aussi sa capacité de disposer ellemême son aporie, le point où elle ne passe plus ${ }^{39}(C M, 203)$.

Il nous sera ainsi montré comment Bouvard et Pécuchet, en radicalisant le projet flaubertien - « [convertir] la bêtise du monde en bêtise de l'art » $(P M, 118)-$

annulent définitivement la différence imperceptible que le style traçait à chaque phrase entre le bavardage de la lettre et son mutisme (PM, 120).

La Parole muette est, de fait, un «Essai sur les contradictions de la littérature », qui entend montrer comment Flaubert, Mallarmé et Proust, loin de les résoudre, embrassent ces contradictions pour en faire le cœur même de leurs œuvres ${ }^{40}$. Par-delà l'ouvrage, on est là devant un trait spécifique de pensée. La Mésentente est le titre de la grande synthèse politique de Rancière, le nom du concept qui désigne l'advenu toujours éphémère de la politique. Mais elle est d'abord, de l'aveu du philosophe ${ }^{41}$, une méthode de lecture: l'examen du texte fondateur d'Aristote définissant l'homme comme « animal politique » produit ainsi ce «jeu » d'où émergera précisément le concept de mésentente ${ }^{42}$.

La mise en scène des pensées par Rancière passe par ce travail de re-figuration. Elle s'accomplit par retour des mêmes références dans des propos par ailleurs très divers. Le jeu de reprise et d'enrichissement au fil des ouvrages constitue en définitive une sorte de répertoire - où coexistent la fable de l'Aventin racontée par Ballanche, les épisodes du Quichotte, les mythes du Phèdre, l'intrigue du Curé de village, etc. -, une petite bibliothèque où ces morceaux se voient redistribués et réarticulés autrement.

38. «Deleuze, Bartleby et la formule littéraire » $(C M, 179-203)$.

39. Il s'agit en l'occurrence du passage établi - via les personnages mythiques d' « originaux » chers à Melville que Deleuze érige en prophètes et prototypes de la grande communauté fraternelle -, mais toujours différé par Deleuze, de la littérature à la politique.

40. Voir l'introduction, « D'une littérature à l'autre », p. 5-14.

41. «The Thinking of Dissensus », réponse aux interventions du colloque Fidelity to Disagreement.

42. Tel est l'objet du «Commencement de la politique» (Més., 19-40). 
B.

Il m'a semblé apprendre, à l'expérience, que la force d'une pensée tenait plutôt à sa capacité d'être déplacée, comme peut-être la force d'une musique à sa capacité d'être jouée sur d'autres instruments que les siens.

La nouvelle configuration donnée au texte trouve son sens dans ce déplacement, en des connexions cette fois externes, où se distinguent à nouveau deux formes de transgression. La transgression temporelle coule de source, elle relève de l'anachronisme déjà rencontré. Ainsi ne s'agit-il pas simplement d'établir des généalogies, ou de faire de Platon ou Flaubert «notre contemporain », au sens où l'on exhiberait la «modernité » de leur pensée. Les contemporanéités ne se révèlent pas, elles se construisent: bien loin d'intégrer un auteur dans « notre » temps - il lui est extérieur autant qu'au sien propre -, la lecture doit le constituer comme intrus.

Le cas Jacotot est exemplaire : penseur égalitaire d'une raison partagée, c'est un homme des Lumières, un homme du XVIII siècle plongé dans le XIX ${ }^{\mathrm{e}}$. Dans Le Maître ignorant, l'effacement du commentateur, l'indistinction calculée entre la voix de Rancière et celle de Jacotot redoublent cette intemporalité au point de faire douter de l'existence même du fondateur de l'enseignement universel. On en vient - la rareté des notes aidant - à le prendre pour une figure fictionnelle forgée dans un but polémique: ses théories émancipatrices bouleversent un débat contemporain sur l'école, elles sapent ses données mêmes ${ }^{43}$. Le présupposé égalitaire de Jacotot renvoie dos à dos des discours - celui des «progressistes », dans le sillage des analyse des Héritiers de Bourdieu et Passeron, celui des "républicains », représentés par l'auteur de $D e$ l'école, Jean-Claude Milner - s'opposant autour d'une conception commune du savoir comme facteur d'émancipation.

La seconde transgression va de pair avec la première, elle est d'ordre disciplinaire. Dans «La surface du design », Rancière déclare employer la méthode des « devinettes enfantines où l'on demande quelle ressemblance ou quelle différence il y a entre deux choses » (DI, 106) et s'attache ensuite à rapprocher Mallarmé de l'ingénieur- 
designer Peter Behrens ${ }^{44}$. Ce jeu, fidèle à sa critique du propre, lui est usuel. Il s'y livre par exemple en interrogeant l'articulation entre l'inconscient freudien et les théories romantiques qui font « de l'art le territoire d'une pensée présente hors d'elle-même, identique à la nonpensée » $(I E, 13)$. Comme il se doit, le lien n'implique aucune subordination d'un domaine à l'autre :

Il s'agit bien plutôt de marquer les rapports de complicité et de conflit qui s'établissent entre l'inconscient esthétique et l'inconscient freudien (IE, 43).

Le dispositif mis en place aménage l'espace où se croisent Freud, Corneille, Hegel, Panofsky, etc. D'autres opérations aussi saisissantes viennent nouer les romans de Flaubert, l'histoire et le cinéma ${ }^{45}$, Rocard, Searle et Don Quichotte ${ }^{46}$, etc. Deux questions se posent, aux intersections de ces exemples. Celle du principe de connexion d'abord: qu'ont en commun ces pensées qui permet de les lier? Celle de la visée ensuite: à quoi tendent ces multiples rapprochements, au-delà du caractère polémique déjà souligné? Il est tentant de faire de la politique le principe rassembleur de tous ces « dispositifs », eu égard au parcours de Rancière, et aux enjeux de son travail. Ce serait simplifier, et méconnaître la spécificité de ce qu'il entend par politique. Il ne s'agit pas de politiser les discours, mais de dégager les figures d'un espace commun qu'ils dessinent. Dans la terminologie de Rancière, un partage du sensible:

J'appelle partage du sensible ce système d'évidences sensibles qui donne à voir en même temps l'existence d'un commun et les découpages qui y définissent les places et les parts respectives $(P S, 12)$.

Plutôt qu'une politique à proprement parler, une esthétique (PS, 1415) que les formes de l'art et de la politique configurent (et défigurent). On a ainsi vu comment la démocratie se définissait comme circulation aléatoire des énoncés. Se précise de la sorte le type de rapport entre art et politique:

44. Autour de l'idée de type entendu comme forme essentielle qui vienne fonder la vie commune $(D I, 111)$.

45. Voir « L'historicité du cinéma », dans De l’histoire au cinéma, 1998.

46. « L'inadmissible », art. cit. 


\section{Politiques de la lecture}

C'est [au niveau] du découpage sensible du commun de la communauté, des formes de sa visibilité et de son aménagement, que se pose la question du rapport esthétique/politique. C'est à partir de là qu'on peut penser les interventions politiques des artistes (PS, 24).

Si l'on a éclairé ici la manière dont s'ajustent les pièces des montages de Rancière, demeure un point aveugle: ce qui permet tel mode de lecture, tels démantèlement, remembrement, et appréhension des œuvres comme dispositifs de pensée.

Mettre en lumière les conditions de possibilités de son travail, c'est précisément sa visée, et la réponse à notre seconde question. Les rapprochements de Rancière tendent à inscrire les œuvres envisagées dans un champ de possibles historiquement situé. Son ouverture a pour nom révolution esthétique, ou romantique.

\section{III - MONTAGES}

\section{La révolution romantique}

La référence au romantisme s'est faite de plus en plus aiguë chez Rancière, au point que l'on peut regarder ses derniers livres (depuis 1998) ${ }^{47}$ comme différentes étapes d'une vaste enquête sur ce qu'il nomme « régime esthétique » de l'art. Rancière retrace en divers textes les contours essentiels de la révolution romantique, acte de naissance dudit régime, en montrant d'abord comment la « littérature » en tant que telle remplace les « belles-lettres »: fin du système représentatif et de sa hiérarchie générique, où correspondent strictement sujets, styles et genres selon leur dignité, où prévaut l'intrigue aristotélicienne, et où la parole en acte tient lieu de paradigme, tout cela entrant « en analogie avec une vision hiérarchique de la communauté » $(P S, 31)$; s'y oppose un régime fondé sur l'égalité esthétique, soit l'indifférence des sujets (et corollairement l'abolition des genres), obéissant à un paradigme de l'écriture, où l'intrigue traditionnelle est supplantée par le « primat du langage »- c'est-à-dire par l'idée que la poésie révèle

\footnotetext{
47. On y ajoutera les articles: « La forme et son esprit », dans La Forme en jeu, 1998; «Y a-t-il un concept du romantisme? ", dans Modernité et romantisme, 2001; "The Aesthetic Revolution and its Outcomes ", dans la New Left Review $\mathrm{n}^{\circ}$ 14, 2002, et les chroniques parues dans les Cahiers $d u$ cinéma entre 1998 et 2001.
} 
une essence du langage, libéré de ses fonctions communicatives. Autrement dit, l'œuvre littéraire n'est plus une représentation mais une manifestation sensible de la pensée (d'où le terme « esthétique », qui désigne cette intensité sensible) détachée des conditions du faire projet et puissance. Et ce qui vaut pour la littérature vaut pour l'art en général, et le «primat du langage » s'élargit en «absoluité du sensible ». L'œuvre esthétique ne se donne qu'à ressentir, c'est-à-dire ni à connaître, ni à désirer. Il n'est donc plus question de définir l'art par les techniques spécifiques d'un faire, une forme s'appliquant à une matière, un actif à un passif. À ceci près que l'activité artistique ne peut se passer de ces couples conceptuels: dès lors, l'œuvre esthétique ne peut être qu'identité des contraires, de l'actif et du passif, du conscient et de l'inconscient; le génie kantien «fait plus qu'il ne veut faire et qu'il ne sait qu'il fait ${ }^{48} »$. Mais surtout, ce régime de l'art ne peut être un nouveau système de normes, puisqu'il révoque l'idée même de normes. En d'autres mots:

Le régime esthétique des arts n'a pas commencé avec des décisions de rupture artistique. Il a commencé avec des décisions de réinterprétation de ce que fait ou de ce qui fait l'art. [...] Le régime esthétique des arts est d'abord un régime nouveau du rapport à l'ancien $(P S, 35)$.

Et c'est à un lecteur, Vico, à la recherche du « véritable Homère », que Rancière attribue le premier geste de la révolution esthétique : récusant la thèse d'une sagesse celée dans les poèmes homériques, il avance l'idée que la poésie n'est que le langage « d'un temps où la pensée ne se séparait pas de l'image » et qu'en conséquence « ses images ne sont rien d'autres que la manière de parler des peuples de son temps » (IE, 29).

Avant d'être l'invention d'une poétique nouvelle, le régime esthétique est une nouvelle façon de lire. En quoi l'on peut dégager la tension fondamentale qui l'habite: si la poésie est un état du langage, alors les œuvres sont la manifestation de cette essence langagière; mais, en vertu du principe d'indifférence, toute forme sensible peut être «lue » de la sorte,

48. « La forme et son esprit », art. cit., p. 140. 


\section{Politiques de la lecture}

toute configuration de propriétés sensibles peut alors être assimilée à un arrangement de signes, donc à une manifestation du langage en son état poétique premier $(P M, 41)$.

Contradiction insoluble entre primat du langage et principe d'indifférence: elle remet en scène la figure double de la parole muette (le langage dépouillé de la garantie d'un sens, réduit à la stricte insignifiance de son être) et bavarde (tout parle, tout est langage). C'est autour d'elle que vont se répartir les options de lecture historiquement attestées. Si l'on privilégie le «primat du langage », on rejouera la fable de l'autotélisme artistique; mais on pourra aussi faire de l'art la révélation d'une pure présence des choses mêmes, lui accorder le privilège de vérités inaccessibles à la raison, et définir la littérature comme pure expérience de l'indicible; voire, en radicalisant encore cette expérience littéraire, la dissoudre jusqu'à son impossibilité même, la folie. À l'inverse, se tourner vers le principe d'indifférence, ce sera rapporter toute œuvre à un principe ludique, pur jeu de l'esprit ${ }^{49}$; selon l'humeur, on déplorera ou on exaltera l'indistinction entre œuvres d'art et marchandises, en quoi le régime esthétique « fonde en même temps l'autonomie de l'art et l'identité de ses formes avec celles par lesquelles la vie se forme elle-même » $(P S, 33)$; enfin l'on dégagera une véritable herméneutique de l'idée - formulée par Novalis (voir $P M, 43$ ) - que « tout parle », un décryptage de ces discours que tiennent les choses du monde: c'est le projet de Balzac, qui donne charge au roman d'interpréter la prolifération de signes qui nous entourent; c'est aussi celui des sciences humaines et sociales qui construisent un savoir sur une société à partir de ce qu'elle exprime. Où, dans une lignée ouverte par l'Homère vichien, réapparaissent Marx, Althusser, Bourdieu, etc.

Ce détour par le romantisme nous ramène à notre chemin. En pointant la contradiction, Rancière entend réaffirmer la complémentarité de ses deux bords, montrer le socle commun aux méditations de Blanchot et aux analyses de Bourdieu (voir PM, 49-52) afin de les renvoyer dos à dos: briser les fictions du propre et les dramaturgies destinales revient à en faire apparaître la solidarité dans le champ ouvert par le régime esthétique. D'où l'établissement de connexions

\footnotetext{
49. Voir « L'artifice, la folie, l'œuvre » $(P M, 141-166)$ où Rancière identifie ces deux positions limi-
} tes, celle de la folie et celle du jeu, aux figures d'Artaud et de Poe. Lire en particulier p. 145-152. 
entre pratiques, œuvres, théories, afin de pourchasser semblablement démystification et sacralisation - et si la détermination de Rancière apparaît plus ferme à combattre la première, c'est affaire de conjoncture, d'un temps qui érige le retour aux « simples pratiques » en sagesse supérieure. La dimension polémique de la lecture est toujours présente, et commande les orientations du philosophe; s'il se préoccupe d'art, à présent, c'est que

le terrain esthétique est aujourd'hui celui où se poursuit une bataille qui porta hier sur les promesses de l'émancipation et les illusions et désillusions de l'histoire $(P S, 8)$.

Poursuivre cette bataille, c'est pour Rancière rester fidèle aux contradictions du régime esthétique, les tenir coûte que coûte, sans privilégier l'un ou l'autre versant.

\section{Les périls de l'entre-deux}

Dès lors, ce régime peut apparaître comme horizon indépassable, foyer de tous les possibles. Survient un doute: si toute œuvre, tout discours s'inscrit dans ce système, Rancière ne retombe-t-il pas dans une nouvelle forme de l'historicisme auquel il prétendait échapper? C'est ce que pressent Laurent Jenny, qui commente l'histoire littéraire déductible de La Parole muette:

Rancière suggère que la littérature n'a pas d'histoire [...] rien ne peut plus lui arriver qui excède ou déplace son concept (ou plutôt son impossibilité conceptuelle). [Elle n'est plus qu'] un simple déploiement formel du système d'impossibilité qui la constitue ${ }^{50}$.

À la linéarité des destins, Rancière substituerait l'éternel mouvement de balancier, guère moins monotone, d'un terme à l'autre de la contradiction. Grave accusation, portée à un penseur de l'événement. Il peut bien sûr répondre par l'argument de non exhaustivité: l'idée de littérature - et par extension l'idée d'art en général - n'épuise pas le sens des œuvres singulières, et ne consiste que comme donnée fondamentale avec laquelle l'œuvre doit jouer; en un mot, une condition des

50. La Fin de l'intériorité, Paris, Puf, coll. « Perspectives littéraires », 2002, p. 11. 


\section{Politiques de la lecture}

œuvres, mais nécessaire ${ }^{51}$. La remarque de Laurent Jenny tend de plus à envisager La Parole muette d'un strict point de vue " littéraire », c'est-à-dire disciplinaire, délaissant un aspect essentiel de l'idée d'art: c'est qu'elle est « d'emblée connectée à une idée de la communauté. [...] [L'esthétique] est la pensée du devenir sensible qui rend les idées communes », autrement dit, l'art " tisse cette étoffe glorieuse du monde commun ${ }^{52} »$. Il ne s'agit pas pour Rancière de retomber dans un autotélisme raffiné, mais d'examiner comment la pensée de l'œuvre, travaillée par cette contradiction première, configure un «partage du sensible ». Il peut alors invoquer à nouveau la nécessité rectificatrice qui contraindrait à toujours défendre la fécondité de la contradiction contre le « ressentiment anti-esthétique ${ }^{53} »$ contemporain, sous forme de synthèses ouvrant la voie aux analyses singulières.

N'en demeure pas moins un trouble devant le non-choix, l'éternel suspens qui signe les interventions de Rancière. Badiou le formule ainsi :

Ses livres ne sont ni des conclusions, ni des directives, mais des clauses d'arrêt. Vous saurez ce que la politique ne doit pas être, vous saurez même ce qu'elle aura été et n'est plus, mais jamais ce qu'elle est dans le réel, et encore moins ce qu'il importe de faire pour qu'elle existe $^{54}$.

Dans une analyse aux termes pas toujours amènes, Badiou pointe du doigt cette « délectation » de Rancière pour l'intervalle et stigmatise son refus de la prescription. Indépendamment du débat entre deux penseurs, il y a là une mise en cause du statut même des discours de Rancière, et de leur portée. La prescription ne peut qu'être étrangère à une réflexion fondée sur un présupposé égalitaire. Badiou fait pourtant de cette posture une «maîtrise ironique de qui prend le maître en défaut ${ }^{55}$ ».

51. Pour une idée de ce que serait une « histoire de la littérature » selon Rancière, il paraît pertinent - et en phase avec sa pratique des connexions - d'interroger ses suggestions en matière d'histoire du cinéma, notamment dans deux entretiens dans les Cahiers du cinéma: « Les mots de l'histoire du cinéma », n 496, novembre 1995, p. 48-54 et « Le cinéma, art contrarié », n 567, avril 2002, p. 56-63.

52. «L'historicité du cinéma », art. cit., p. 55-56.

53. Titre d'un article paru dans Le Magazine littéraire, novembre 2002.

54. Abrégé de métapolitique, op. cit., p. 125.

55. Ibid., p. 121-122 
Se profile alors une ultime figure du rapport à la lecture, après celle de l'objet de réflexion et celle de la pratique, celle des relations de Rancière avec ses propres lecteurs ou: comment être lu? Se greffe ici une dernière réserve, celle d'Yves Michaud, qui s'interroge précisément sur les destinataires de Rancière:

Le style même des interventions de Rancière, elliptiques, cultivées, ironiques, exprimées dans un langage hautement philosophique, oblige à demander à qui il s'adresse. Si c'est à sa communauté intellectuelle (philosophique), gageons qu'elle risque peu de l'entendre. [...] Si c'est à d'autres, à qui donc ${ }^{56}$ ?

\section{Lire Jacques Rancière}

Soient trois estimations critiques du travail de Rancière dont les origines, les perspectives et les tons divergent sensiblement, mais qui se rejoignent dans une interrogation commune sur les effets de cette entreprise. Ce faisant, ces trois points de vue n'envisagent pas - sciemment ou non, à tort ou à raison, là n'est pas le propos - la pensée de Rancière à l'aune de ses propres analyses du régime esthétique, contraires à toute idée d'un effet prédéterminé du discours et d'une stabilité de son statut. Précisons : Jenny fait de l'impropre littéraire un pur substitut de l'essence; Badiou ignore le refus égalitaire de prescrire; Michaud voit dans le refus illusoire de s'adresser à un public défini une dénégation du véritable public visé, la communauté des pairs: réfutation sociologique de la parole errante. Je n'entends pas discuter le bien-fondé de ces remarques, mais essayer d'approcher leur cible par un autre biais, en considérant Rancière dans l'univers conceptuel qu'il explore. Dit autrement: quel statut donner à sa philosophie, et quels effets politiques lui prêter, en tant que configuration singulière des possibles ouverts par le régime esthétique?

À une question sur la distance relative qu'il ménage à l'égard des débats politiques contemporains, Rancière répond: « Ce que je peux apporter à la politique, c'est une certaine reconfiguration des données et des problèmes ${ }^{57}$. » Le philosophe se fait metteur en scène, figure déjà rencontrée à propos du « rephrasage » des auteurs. La métaphore de

56. « Les pauvres et leur philosophe », dans Critique, $\mathrm{n}^{\circ}$ 601-602, « Autour de Jacques Rancière », juin-juillet 1997, p. 443.

57. « Le Maître ignorant - entretien avec Jacques Rancière », Vacarme, n ${ }^{9}$, automne 1999, p. 5. 


\section{Politiques de la lecture}

la scène est capitale ${ }^{58}$ dans une réflexion politique qui définit la démocratie comme jeu d'apparences:

Il y a démocratie s'il y a une sphère spécifique d'apparence du peuple. [...] Il y a démocratie s'il y a des acteurs spécifiques de la politique. [...] Il y a démocratie enfin s'il y a un litige construit sur la scène de manifestation du peuple par un sujet non identitaire ${ }^{59}$ (Més., 141).

Métaphore théâtrale? En partie, mais pas seulement:

Il se trouve en effet que la « mise en scène » n'est aucunement un art auxiliaire du théâtre, dépendant de la tradition représentative. Elle est un art à part entière, un art propre à l'âge esthétique ${ }^{60}$.

Dès lors, la scène ne concerne pas que le théâtre, mais aussi le cinéma, qui joue pour Rancière un rôle décisif: il est l'art esthétique par excellence, « un art dont le concept existait déjà par avance, un siècle avant la première grande séance publique de Pathé ${ }^{61}$. » Celui qui, d'après des théoriciens comme Epstein ou Canudo, permettrait enfin cette épiphanie du sensible en sa pure vérité - la « bêtise » flaubertienne des chefsd'œuvre - par l'identité des contraires ${ }^{62}$ : le processus inconscient (enregistrement mécanique de la caméra) et la démarche consciente (l'œil et la main de l'homme). Mais surtout, la logique esthétique se marque dans la dimension de l'après-coup, c'est-à-dire dans le démantèlement des intrigues de l'art représentatif; «tel est l'art de l'âge esthétique: un art qui vient après et défait les enchaînements de l'art représentatif » $(F C, 16)$. Plus manifestement encore que d'autres, le

58. Pierre Campion met en évidence cette notion de scène, et ses implications quant au rapport entre littérature et politique dans «Littérature et politique. Compte rendu du Partage du sensible de Jacques Rancière », Acta Fabula, http://www.fabula.org/revue/cf/17.php

59. Voir également le titre donné au recueil des articles parus dans Les Révoltes logiques: Les Scènes du peuple.

60. «L'historicité du cinéma », art. cit., p. 58.

61. Ibid., p. 53.

62. En ce point se manifeste la singularité de l'art cinématographique, par la forme spécifique que prend chez lui la contrariété esthétique: elle se complique d'une passivité première, et non gagnée par le travail de l'artiste. Contrarier sa «pureté » originelle, c'est donc justement pour le cinéma perturber cette passivité. Pour la démonstration complète de cette appartenance de l'art cinématographique au régime esthétique, et ses conséquences dans les œuvres singulières, voir $F C$, (en particulier le prologue «Une fable contrariée »), ainsi que « L'historicité du cinéma », art. cit. 
cinéma est art de lecture, c'est-à-dire de montage - une pratique et une notion qu'il aurait empruntées aux romanciers du XIX ${ }^{e}$ siècle $(D I, 58)$ :

Un travail de dé-figuration [...], [celui] que proposaient à l'aube du $\left[\mathrm{XIX}^{\circ}\right]$ siècle les textes de l'Athenäum des frères Schlegel, au titre de la fragmentation romantique qui défait les anciens poèmes pour en faire les germes de poèmes nouveaux $(F C, 15)$.

Mais ce travail a aussi à voir avec celui de Jacques Rancière, qui retrouve le même mot de " dé-figuration » pour qualifier sa propre lecture de Deleuze, déjà évoquée. S'il ne s'agit pas pour lui de défaire "les assemblages de la fiction ou du tableau représentatif », c'est bien d'un processus de montage qu'il s'agit, qui « re-voit, re-lit et re-dispose les éléments » $(F C, 15)$ d'une pensée.

Je m'autoriserai donc, pour spécifier le statut de son travail et ses effets possibles, à user d'une notion par lui forgée, celle de «phraseimage $^{63} »$. À savoir une procédure de synthèse commune à toutes les formes de l'art esthétique,

qui peut s'exprimer en phrases de roman mais aussi en formes de mise en scène théâtrale ou de montage cinématographique ou en rapport du dit et du non-dit d'une photographie $(D I, 56)$.

La phrase peut fort bien être visuelle, et l'image verbale. L'important est que la première signifie un continuum, la grande contingence de toutes choses, la «bêtise » flaubertienne, en un mot l'effectuation du principe d'indifférence; l'image, elle, désigne la rupture, le choc, l'éclair disruptif qui découle du principe d'absoluité. La phrase-image est donc la contradiction esthétique en acte. Après l'avoir mise en évidence chez Zola, dans un extrait du Ventre de Paris (DI, 58-60), Rancière la confronte aux Histoire( $s$ ) du cinéma, de Jean-Luc Godard ${ }^{64}$, à propos desquelles il en distingue deux usages. L'un, qu'il nomme « montage dialectique », privilégie le pôle de l'image, en affirmant la rupture:

La puissance de la phrase-image qui joint les hétérogènes est alors celle de l'écart et d'un heurt qui révèle le secret d'un monde.

63. La notion est élaborée et travaillée dans « La phrase, l'image, l'histoire », art. cit. (DI, 56 sqq.). 64. Lire aussi «Une fable sans morale: Godard, le cinéma, les histoires » $(F C, 217-237)$. 


\section{Politiques de la lecture}

Il appelle l'autre «montage symboliste », qui tend vers le pôle phrastique, s'employant à

établir une familiarité, une analogie occasionnelle, témoignant d'une relation plus fondamentale de co-appartenance, d'un monde commun où les hétérogènes sont pris dans un même tissu essentiel $(D I, 66-67)$

Godard aurait délaissé la phrase-image dialectique, triomphante « à l'âge des provocations pop » (voir Pierrot le fou), pour la phrase-image symboliste, qui dominerait dans les Histoire( $s$ ). Il illustrerait alors une tendance actuelle de l'art, glissant des dispositifs de dénonciation aux célébrations insolites de la communauté (voir DI, 68-78).

C'est selon cette même mesure de la phrase-image que je veux lire les «montages » philosophiques de Rancière. Au pôle dialectique se place le travail de déliaison, de dissolution des destins en tous genres. Pour estimer son effet, il faut comprendre comment ce travail s'articule avec l'acte politique, l'irruption de la scène égalitaire - qui tient clairement de cette même puissance de l'image: le discours philosophique semblerait se manifester comme un dissensus spécifique, distinct des deux autres, celui qui ressort de la politique proprement dite et celui que met en œuvre la littérature. Le dernier attaquerait le consensus sur un autre flanc, sans se nouer simplement à la subjectivation politique: affaire de singularités, plutôt que de collectivités - pour aller vite -, il ne pourrait fournir le fondement nécessaire à l'élaboration de la scène du litige. Mais il aurait aussi un autre rôle: celui de modèle, sinon pour les subjectivations politiques, du moins justement pour une philosophie égalitaire. Si Rancière ne formule pas explicitement dans ses analyses cette fonction exemplaire des œuvres, il revient à maintes reprises sur les «modèles » qu'offrent à son écriture les romans de Woolf ou Joyce, plus adéquats, justement, à rendre compte de l'événement politique que le réalisme romanesque traditionnel:

Choisir un mode de récit qui ne commence pas par situer, par enraciner, mais qui parte du caractère fragmentaire, lacunaire, indécidable, partiellement décidable de ces paroles [celles des prolétaires], un type de récit à la Virginia Woolf, où il y a des voix qui petit à petit s'entrecroisent et construisent en quelque chose l'espace de leur effectivitét ${ }^{65}$.

65. « Histoire des mots, mots de l'histoire », art. cit., p. 88. 
Ce point d'indistinction entre littérature et philosophie pourrait être source de discussions. Retenons simplement que l'on ne peut confondre tout à fait ces pratiques discursives: le dissensus philosophique s'attaquerait au consensus sur un troisième front, en l'occurrence le domaine des savoirs. Sa cible spécifique serait l'épistémocratie (gouvernement des experts), pendant de son contraire l'ochlocratie (gouvernement de l'opinion) dans l'édifice contemporain de nos postdémocraties ${ }^{66}$. Ainsi la philosophie - celle de Rancière - est une délégitimation du savoir, un «travail de sabotage visant à le rendre malpropre à la consommation et inutile à la domination ${ }^{67}$ », par la mise en scène de la contingence égalitaire. On peut étendre la portée de l'expression «poétique de savoir » au-delà de l'ouvrage qu'elle sous-titre (Les Mots de l'histoire), comme effort pour ramener des pratiques de savoir à des opérations poétiques sur le langage commun: montrer que « la folie utopique représente un infime excès sur les procédures par lesquelles les savoirs positifs et les politiques raisonnables construisent la réalité » $(C V, 10-11)$. Pour revenir à la critique de Michaud, Rancière s'adresse $e t$ ne s'adresse pas aux « communautés scientifiques », cibles autant qu'interlocuteurs. Fragiliser le consensus dans l'ordre du savoir, peut-être serait-ce aussi fournir des instruments de lutte aux subjectivations politiques émergentes, en quoi il y aurait dans cette entreprise quelque chose de la « boîte à outils » théorique de Foucault et Deleuze ${ }^{68}$.

Mais ce versant dissensuel - largement privilégié par Rancière - a son pendant «symboliste ». C'est lui qui est mis en cause par Laurent Jenny, à travers le grand réseau d'équivalences que construit Rancière dans et par le régime esthétique - variante de la guirlande schlégélienne (voir PM, 59). Les Histoire(s) du cinéma, et l'art contemporain avec

66. Toute cette terminologie est déployée dans « La fin de la politique ou l'utopie réaliste » ( $A B, 71$ -

72). Sur l'okhlos, emprunté à La Politique d'Aristote, « foule », rassemblement compact opposé au dèmos (conçu comme puissance de division de l'okhlos), lire p. 43 et 65-73.

67. «La pensée d'ailleurs », Critique, n 369, février 1978, p. 245. Dans ce texte, Rancière va jusqu'à qualifier son entreprise d' « anti-philosophique ». C'est à ma connaissance la seule occurrence du terme - même si Badiou désigne sans nuances cette pensée comme « une antiphilosophie démocratique », Abrégé de métapolitique, op. cit., p. 130.

68. «Les intellectuels et le pouvoir: entretien Michel Foucault-Gilles Deleuze », L'Arc n ${ }^{\circ} 49$, « Gilles Deleuze », 1972, p. 3-10 (en part. p. 5.). Repris dans Foucault, Dits et écrits II, Paris, Gallimard, 1994 , p. $306-316$. 
elles, s'attacheraient aujourd'hui à « constituer le monde des « images » comme monde de la co-appartenance et de l'entre-expression généralisées » $(D I, 73)$. Chez Rancière, on vu aussi, succédant aux mouvements de déliaisons, ces fragiles rattachements par liens inconsistants. Qu'elle soit fantomatique ou contingente, il y a bien ici la figuration d'une communauté. Peut-être pourrait-on ainsi comprendre une formule parfois employée par Rancière pour évoquer son travail, celle de « cartographie des possibles ${ }^{69}$ ». Ou l'idée d'une patiente transcription de données, de chemins multiples esquissés; mais aussi celle du territoire inconnu, que l'on rend étranger - ou face auquel on devient soi-même étranger:

L'étranger [...] déplace son angle, retravaille le montage premier des mots et des images, défaisant les certitudes du lieu, réveille le pouvoir présent en chacun de devenir étranger à la carte des lieux et des trajets généralement connue sous le nom de réalité ( $C V, 9-10)$.

La carte est bien un montage: version alternative de la "réalité », partielle, approximative, elle suscite une nouvelle et dernière référence à un terme foucaldien - qui marque encore un peu plus la dette de Rancière à l'auteur des Mots et les choses $^{70}-$, l'hérétopie $^{71}$. Remise au goût du jour par Rancière ( $P S, 64-65)$, elle lui permet surtout de révoquer l'utopie comme rêve de consistance, de communauté incorporée ${ }^{72}$. On risquera aussi une double définition de l'autre qu'elle implique: comme autre du consensus, qui vient brouiller ses évidences; mais peut-être aussi comme l'autre à qui elle s'adresse, vers qui elle tend.

69. Difficile, à ce mot, de ne pas faire allusion de nouveau à celui dont Deleuze avait fait un « nouveau cartographe », Michel Foucault (« écrivain non: un nouveau cartographe », à propos de Surveiller et punir, dans Critique, $\mathrm{n}^{\circ}$ 343, décembre 1975, p. 1207-1227). Les remarques de Deleuze sur la notion de diagramme (Ibid., p. 1223), inspirées par le fameux chapitre sur le «Panopticon », font écho à la représentation de la « réalité », et aux traitements à lui infliger, chez Rancière - et inscrivent peut-être d'autant plus ce dernier dans la généalogie foucaldienne.

70. Il faudrait préciser: Badiou note justement l'absence du «postulat nietzschéen » chez Rancière (Abrégé de métapolitique, op. cit., p. 121); ce dernier par ailleurs marque à la fois sa proximité et sa distance d'avec Foucault dans La Mésentente, principalement par son refus de mettre le concept de pouvoir au centre de la réflexion sur la politique.

71. Voir Michel Foucault, «Des espaces autres », (conférence au Cercle d'études architecturales, 14 mars 1967), publié dans Architecture, Mouvement, Continuité, n 5, octobre 1984, p. 46-49, repris dans Dits et écrits IV, Paris, Gallimard, 1994, p. 752-762.

72. Voir « Sens et usages de l'utopie », dans L'Utopie en questions, 2001, p. 65-78. 
La carte ne représente pas une fin en soi, elle appelle l'usage et les révisions : le personnage du cartographe, intermédiaire entre les découvreurs d'un territoire et les futurs arrivants, s'accorde bien avec une philosophie de l'après-coup, ou plutôt de la continuation, qui refuse tout autant les déclarations tapageuses de table rase que les discours crépusculaires. Incarnation d'un relais, il passe le témoin à d'hypothétiques autres.

À ceux-ci tout loisir est laissé d'éplucher la carte, de n'en considérer qu'une partie, de la gribouiller ou tout simplement de la garder dans leur poche. "Peut-être vaut-il mieux effectivement écrire sans destinataire » $(C M, 176)$, telle était l'une des conclusions du regard rétrospectif porté sur l'œuvre d'Althusser. Tel est sans doute aussi le postulat des fictions hétérotopiques et philosophiques de Jacques Rancière, qui pourtant doivent parier sur l'attention de l'autre, et sur la possibilité de la communauté. Toute aventure comporte ses risques, pas moins les aventures intellectuelles que dessinent les livres:

Or il y a mille manières de traiter un livre - de le lire et de se l'approprier: comme passe-temps et comme objet de culte; comme démonstration rigoureuse ou comme histoire fabuleuse, comme Bible ou comme Robinson Crusoé; en jouissant de ses attentes différées ou en le commençant par la fin; en le prenant au propre ou au figuré; en $y$ croyant ou en n'y croyant pas; en n'y croyant pas et en y croyant. L'usage d'un livre est fait de la combinaison variable de ces choix qui peuvent aussi se dépouiller de leur caractère alternatif. C'est de cette manière qu'on l'insère dans l'écriture de sa propre vie comme dans la refiguration d'un partage commun du sensible ${ }^{73}$.

Défendre l'insularité livresque, et les communautés inconsistantes qui en procèdent; défigurer des dispositifs de pensées, et les re-figurer en un vaste réseau de références; fissurer l'édifice consensuel et cartographier les possibles communautaires. La lecture sous toutes ses formes nous a entraînés dans le geste double de la rupture et du rassemblement. Badiou distinguait chez Rancière une théorie des noms ${ }^{74}$. Nous dirions plutôt une théorie et une pratique de la phrase. Un simple mot, le plus simple qui soit, en figurerait la logique. Rancière le met 


\section{Politiques de la lecture}

en exergue dans la quinzième des Lettres sur l'éducation esthétique de l'humanité, de Schiller, entre les deux bords (art, vie) de la contradiction esthétique ${ }^{75}$ : le mot et. Mot de liaison, de la communauté; mot de déliaison, de séparation, en l'usage que Proust analyse chez Flaubert ${ }^{76}$. Conjonction ou disjonction. Disjonction et conjonction.

75. «The Aesthetic Revolution and its Outcomes », New Left Review, n 14, mars-avril 2002.

76. Marcel Proust, « À propos du "style" de Flaubert » (Nouvelle Revue française, 1 ${ }^{\mathrm{er}}$ janvier 1920), dans Euvres complètes, Contre Sainte-Beuve, Paris, Gallimard, coll. « Bibliothèque de la Pléiade », 1971 , p. 586-600. 